\title{
Use of a Chiral Praseodymium Shift Reagent in Predicting the Complete Stereostructure of Glisoprenin A
}

\author{
Indranath Ghosh ${ }^{\mathrm{a}}$, Yoshito Kishi ${ }^{\mathrm{a}, *}$, Hiroshi Tomoda ${ }^{\mathrm{b}}$, and Satoshi Omura ${ }^{\mathrm{b}}$ \\ ${ }^{a}$ Department of Chemistry and Chemical Biology, Harvard University, \\ Oxford Street, Cambridge, Massachusetts 02138, U.S.A. \\ ${ }^{b}$ Kitasato Institute for Life Sciences, Kitasato University, Minato-ku, Tokyo 108-8641, Japan
}

\section{Preparation of Chiral Shift Reagents}

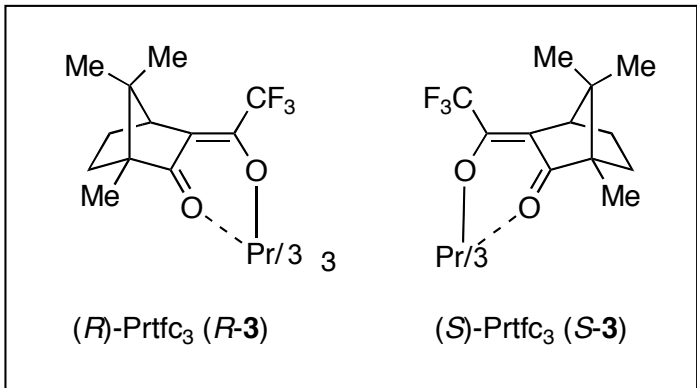

See Supporting Information of preceding Letter.

\section{Preparation of NMR Sample and Recovery of Natural Product}

For the case of glisoprenin A (1) both $(R)$ - and $(S)-\operatorname{Pr}(\mathrm{tfc})_{3}(\mathbf{3})$ were dried at $100{ }^{\circ} \mathrm{C}$ under high vacuum for $36 \mathrm{~h}$ prior to use. Samples were prepared at a concentration of $0.02 \mathrm{M}$ in a mixture of $\mathrm{CD}_{2} \mathrm{Cl}_{2}: \mathrm{C}_{6} \mathrm{D}_{6}$ (v/v 1:4) containing $20 \mathrm{~mol} \%$ per $\mathrm{OH}$ of chiral shift reagent (CSR). The sample prepared was transferred to an NMR tube (that had previously been oven-/vacuum-dried and flushed with argon) via syringe and the tube sealed with teflon tape. Following acquisition, the natural product was recovered by filtration, first through a pipet column of silica gel $(4 \mathrm{~cm}$ tall $)$ and then through a pipet column of basic alumina $(4 \mathrm{~cm}$ tall). For both steps a solvent gradient was employed $\left(\mathrm{CH}_{2} \mathrm{Cl}_{2} \rightarrow 2.5 \% \mathrm{MeOH}\right.$ in $\mathrm{CH}_{2} \mathrm{Cl}_{2} \rightarrow 5 \% \mathrm{MeOH}$ in $\left.\mathrm{CH}_{2} \mathrm{Cl}_{2}\right)$.

\section{Data Acquisition Parameters}

${ }^{1}$ H-NMR: A Varian Inova 500 spectrometer (500 MHz) was used to collect data. The recycle/relaxation delay (d1) was adjusted to $0.1 \mathrm{~s}$ and the experiment was acquired with 16 transients $(\mathrm{nt}=16)$. The readout of NMR spectra was adjusted to $0.001 \mathrm{ppm} / \mathrm{point}(\mathrm{sw}=15000, \mathrm{fn}$ $=524288)$.

${ }^{13}$ C-NMR: A Varian Inova 500 spectrometer $(500 \mathrm{MHz})$ was used to collect data. The recycle delay (d1) was adjusted to $0.0 \mathrm{~s}$ and the experiment was acquired with 30,000 transients $(\mathrm{nt}=30,000)$. The readout of NMR spectra was adjusted to $0.001 \mathrm{ppm} / \mathrm{point}(\mathrm{sw}=29996, \mathrm{fn}=$ 524288).

${ }^{1} \mathbf{H}-{ }^{1} \mathbf{H}$ COSY for $S$-3: A Varian Inova 500 spectrometer (500 MHz) was used to collect data. The recycle delay (d1) was set to $0.2 \mathrm{~s}$ and the experiment was acquired with 256 increments (ni=256) with 148 scans $(\mathrm{nt}=148)$ per increment. The ${ }^{1} \mathrm{H}$ spectral window was adjusted to $5595 \mathrm{~Hz}$ 
$(\mathrm{sw}=\mathrm{sw} 1=5595 \mathrm{~Hz})$ with 2048 data points $(\mathrm{np}=2048)$ and zero-filled to $4096(\mathrm{fn}=4096) . F_{l}$ was linearly predicted to 512 and zero filled to $4096(\mathrm{fn} 1=4096)$. Both $F_{1}$ and $F_{2}$ were processed with sine-bell apodization $(\mathrm{sb}=0.07, \mathrm{sb} 1=0.019)$.

${ }^{1} \mathbf{H}-{ }^{1} \mathbf{H}$ COSY for $R$-3: A Varian Inova 500 spectrometer $(500 \mathrm{MHz})$ was used to collect data. The recycle delay (d1) was adjusted to $0.21 \mathrm{~s}$ and the experiment was acquired with 256 increments $(\mathrm{ni}=256)$ with 148 scans $(\mathrm{nt}=148)$ per increment. The ${ }^{1} \mathrm{H}$ spectral window was adjusted to 5803 $\mathrm{Hz}(\mathrm{sw}=\mathrm{sw} 1=5803 \mathrm{~Hz})$ with 2048 data points $(\mathrm{np}=2048)$ and zero-filled to $4096(\mathrm{fn}=4096) . F_{l}$ was linearly predicted to 512 and zero filled to 4096 (fn1=4096). Both $F_{l}$ and $F_{2}$ were processed with sine-bell apodization $(\mathrm{sb}=0.07, \mathrm{sb} 1=0.019)$.

${ }^{13}$ C-H HSQC for $S$-3: A Varian Inova 500 spectrometer $(500 \mathrm{MHz}$ ) was used to collect data. The recycle delay (d1) was adjusted to $0.3 \mathrm{~s}$ and the experiment was acquired with 512 increments $(\mathrm{ni}=512)$ with 120 scans $(\mathrm{nt}=120)$ per increment. The INEPT transfer delay was shortened to 1.7 ms $(\mathrm{j} 1 \mathrm{xh}=145 \mathrm{~Hz})$, and both sspul and nullflg were set to ' $\mathrm{n}$ '. In the case of using the multiplicity-edited HSQC pulse sequence, the term 'mult' should be changed to 0 . The ${ }^{1} \mathrm{H}$ spectral window was adjusted to $5595 \mathrm{~Hz}(\mathrm{sw}=5595 \mathrm{~Hz})$ with 2048 data points $(\mathrm{np}=2048)$ and zero-filled to 2048 ( $\mathrm{fn}=2048$ ). The ${ }^{13} \mathrm{C}$ spectral window was adjusted to $5278 \mathrm{~Hz}(\mathrm{sw} 1=5278 \mathrm{~Hz}$ ) with $2 \times$ 512 increments linearly predicted to 2048 and zero-filled to $8192(\mathrm{fn} 1=8192)$. Both $F_{1}$ and $F_{2}$ were processed with Gaussian apodization $(\mathrm{gf}=0.03$, $\mathrm{gf} 1=0.1)$.

${ }^{13}$ C-H HSQC for $R$-3: A Varian Inova 500 spectrometer (500 MHz) was used to collect data. The recycle delay (d1) was adjusted to $0.23 \mathrm{~s}$ and the experiment was acquired with 512 increments $(\mathrm{ni}=512)$ with 128 scans $(\mathrm{nt}=120)$ per increment. The INEPT transfer delay was shortened to 1.7 ms $(\mathrm{j} 1 \mathrm{xh}=145 \mathrm{~Hz})$, and both sspul and nullflg were set to ' $\mathrm{n}$ '. In the case of using the multiplicity-edited HSQC pulse sequence, the term 'mult' should be changed to 0 . The ${ }^{1} \mathrm{H}$ spectral window was adjusted to $5803 \mathrm{~Hz}(\mathrm{sw}=5803 \mathrm{~Hz})$ with 2048 data points $(\mathrm{np}=2048)$ and zero-filled to 2048 ( $\mathrm{fn}=2048)$. The ${ }^{13} \mathrm{C}$ spectral window was adjusted to $5529 \mathrm{~Hz}(\mathrm{sw} 1=5529 \mathrm{~Hz})$ with $2 \times$ 512 increments linearly predicted to 2048 and zero-filled to $8192(\mathrm{fn} 1=8192)$. Both $F_{1}$ and $F_{2}$ were processed with Gaussian apodization $(\mathrm{gf}=0.03$, $\mathrm{gf} 1=0.08)$.

HSQMBC: A Varian Inova 500 spectrometer $(500 \mathrm{MHz})$ was used to collect data. The HSQC pulse sequence was employed with the following changes. The recycle delay (d1) was set to 0.35 $\mathrm{s}$ and the experiment was acquired with 128 increments $(\mathrm{ni}=128)$ with 192 scans $(\mathrm{nt}=120)$ per increment. The INEPT transfer delay was changed to $22.7 \mathrm{~ms}(\mathrm{j} 1 \mathrm{xh}=11 \mathrm{~Hz})$, and both sspul and nullflg were set to ' $n$ '. In the case of using the multiplicity-edited HSQC pulse sequence, the term 'mult' should be changed to 0 . The ${ }^{1} \mathrm{H}$ spectral window was adjusted to $5997 \mathrm{~Hz}(\mathrm{sw}=5997 \mathrm{~Hz})$ with 2048 data points $(\mathrm{np}=2048)$ and zero-filled to $2048(\mathrm{fn}=2048)$. The ${ }^{13} \mathrm{C}$ spectral window was adjusted to $1257 \mathrm{~Hz}(\mathrm{sw} 1=1257 \mathrm{~Hz})$ with $2 \times 128$ increments linearly predicted to 1024 and zerofilled to 8192 (fn1=8192). Both $F_{1}$ and $F_{2}$ were processed with Gaussian apodization $(\mathrm{gf}=0.03$, gf1=0.07).

\section{Selected NMR Spectra}



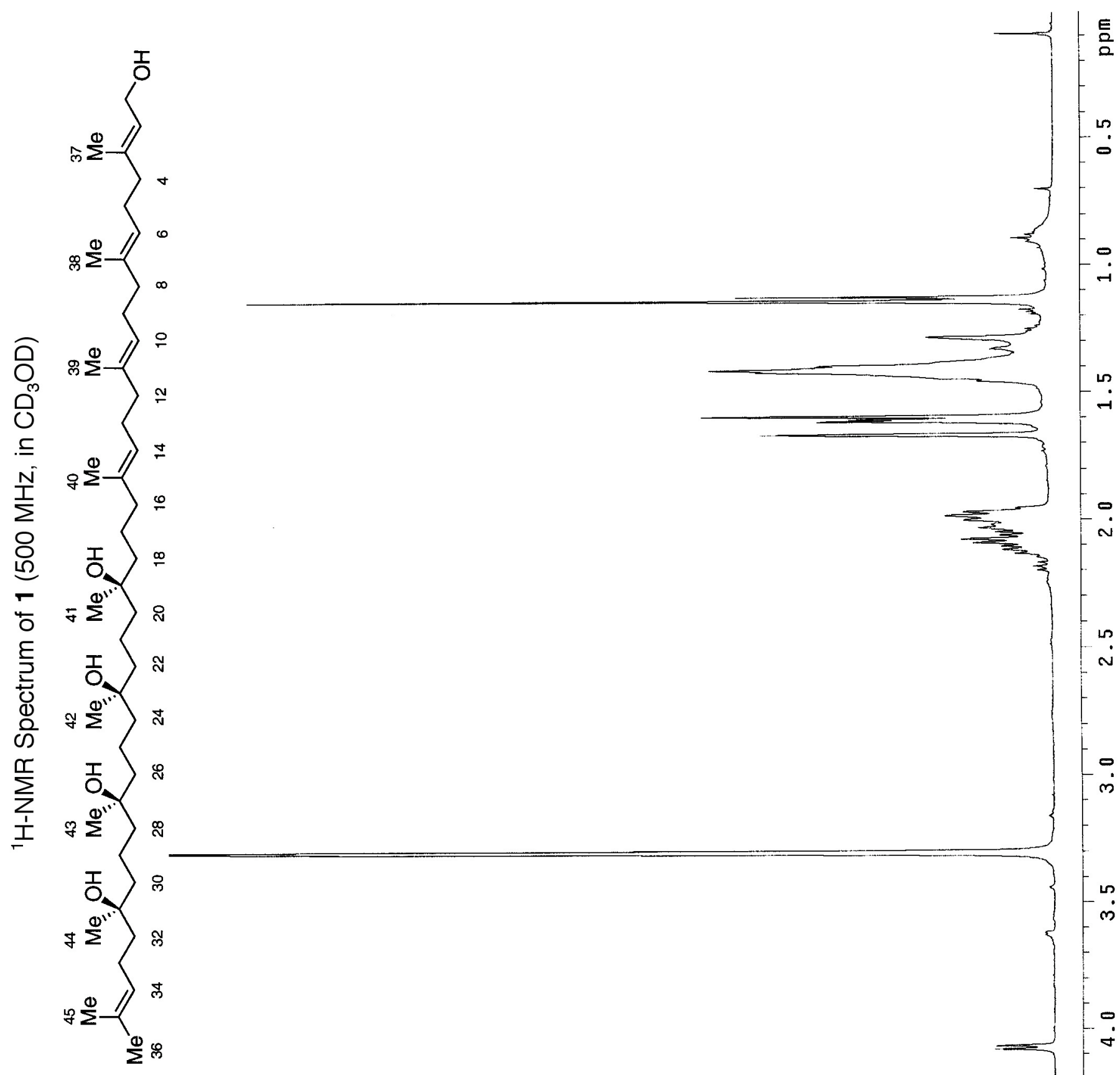

$\frac{1}{2}$
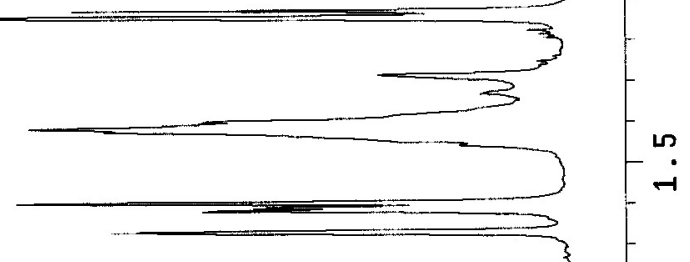


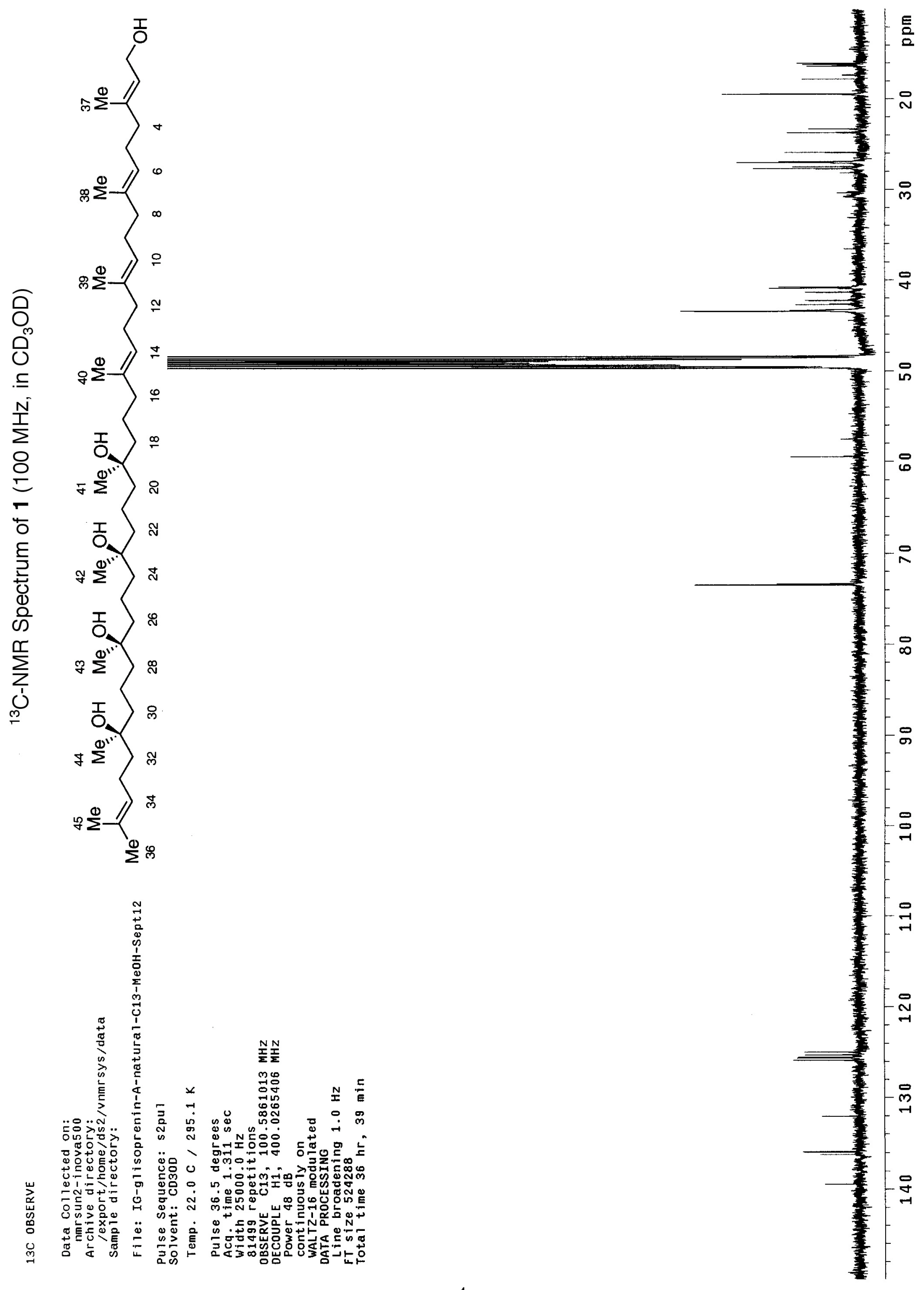



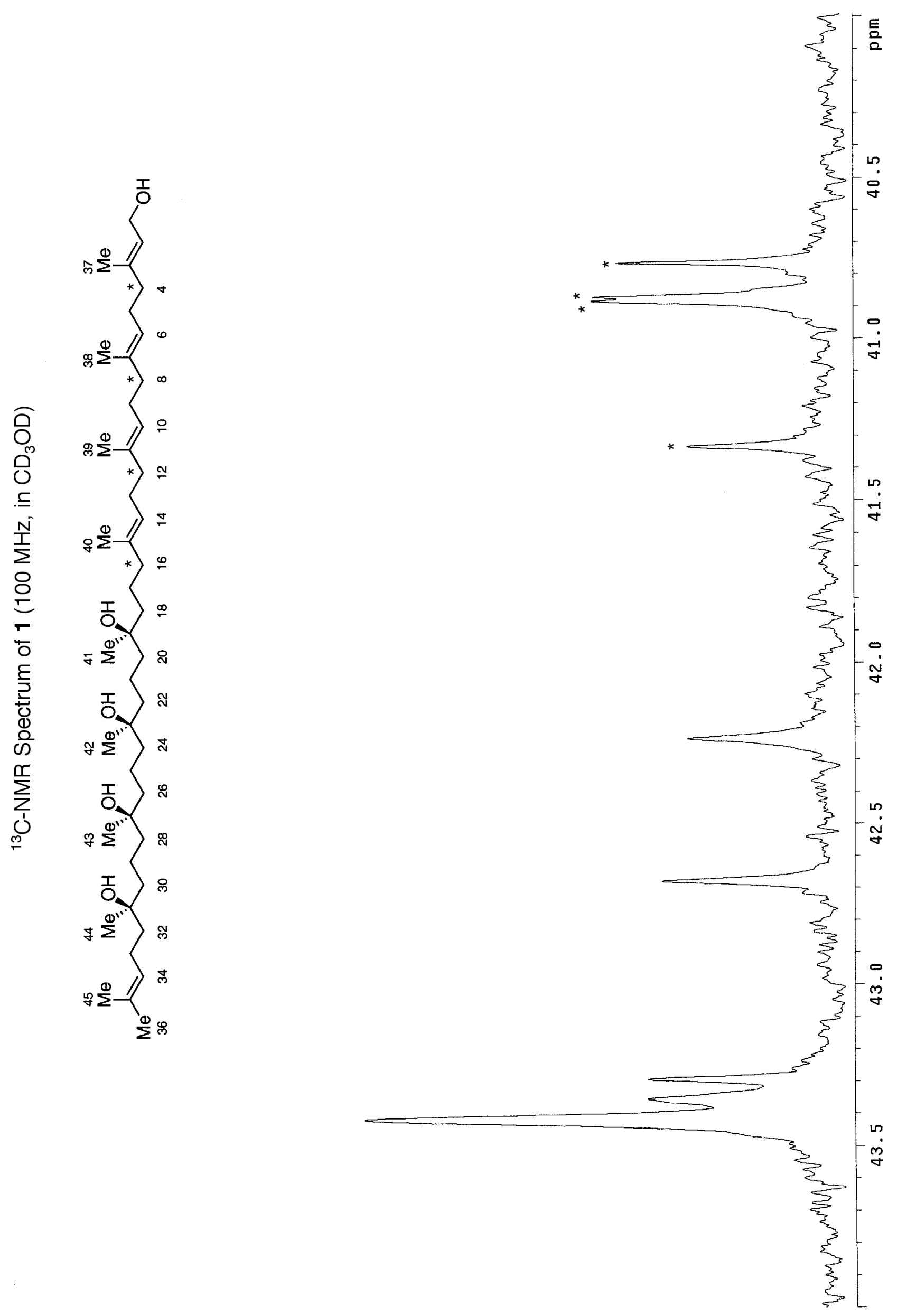


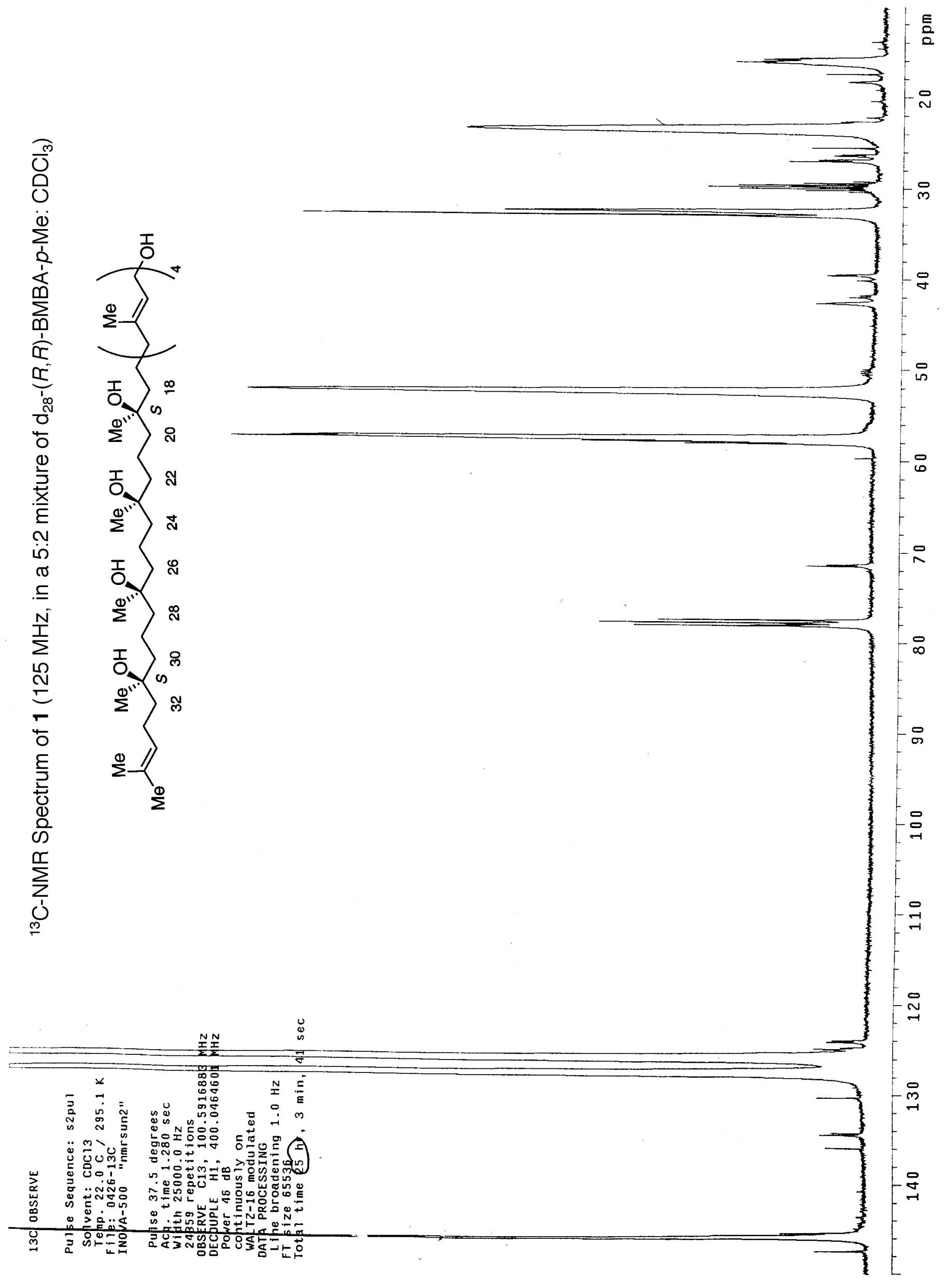



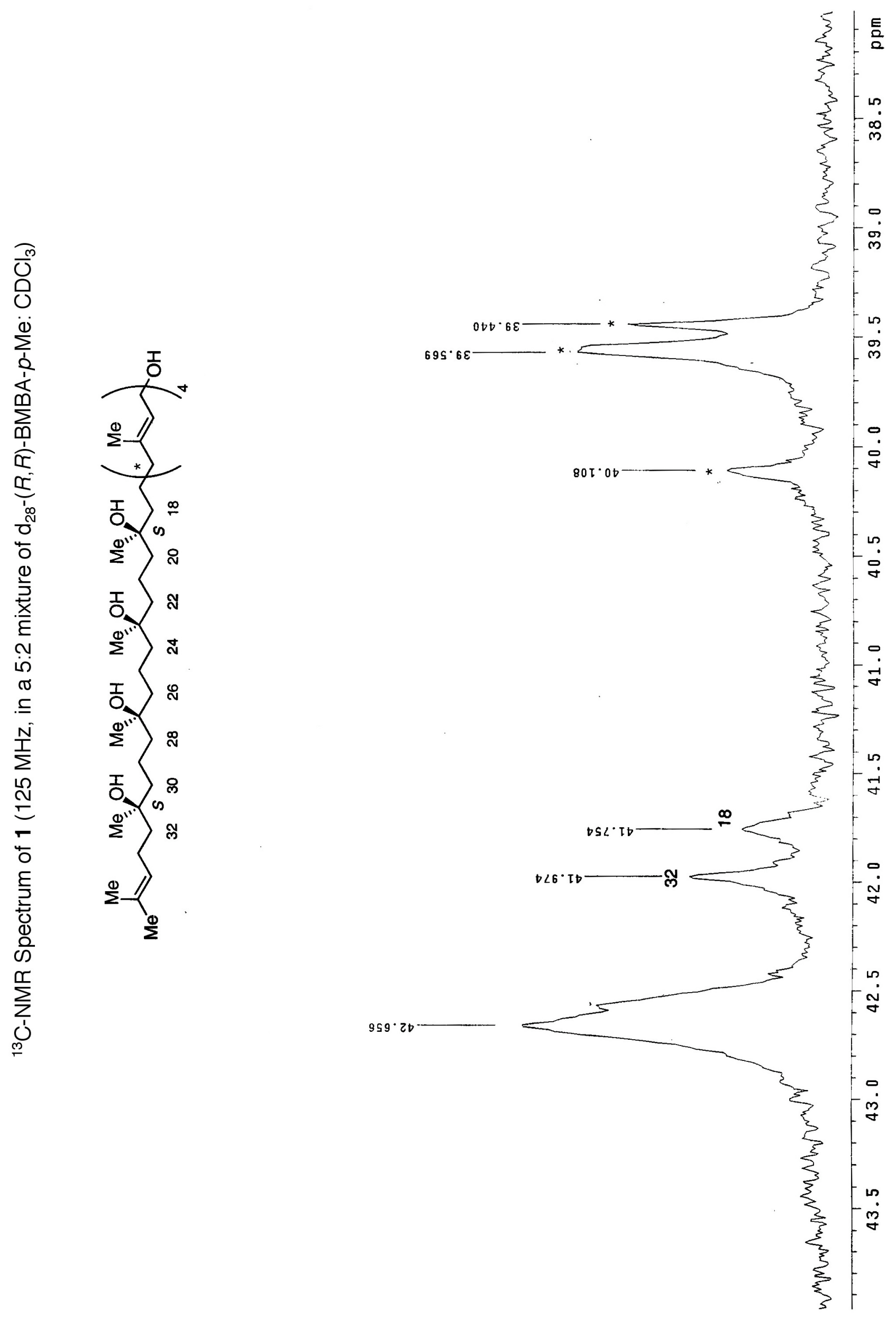


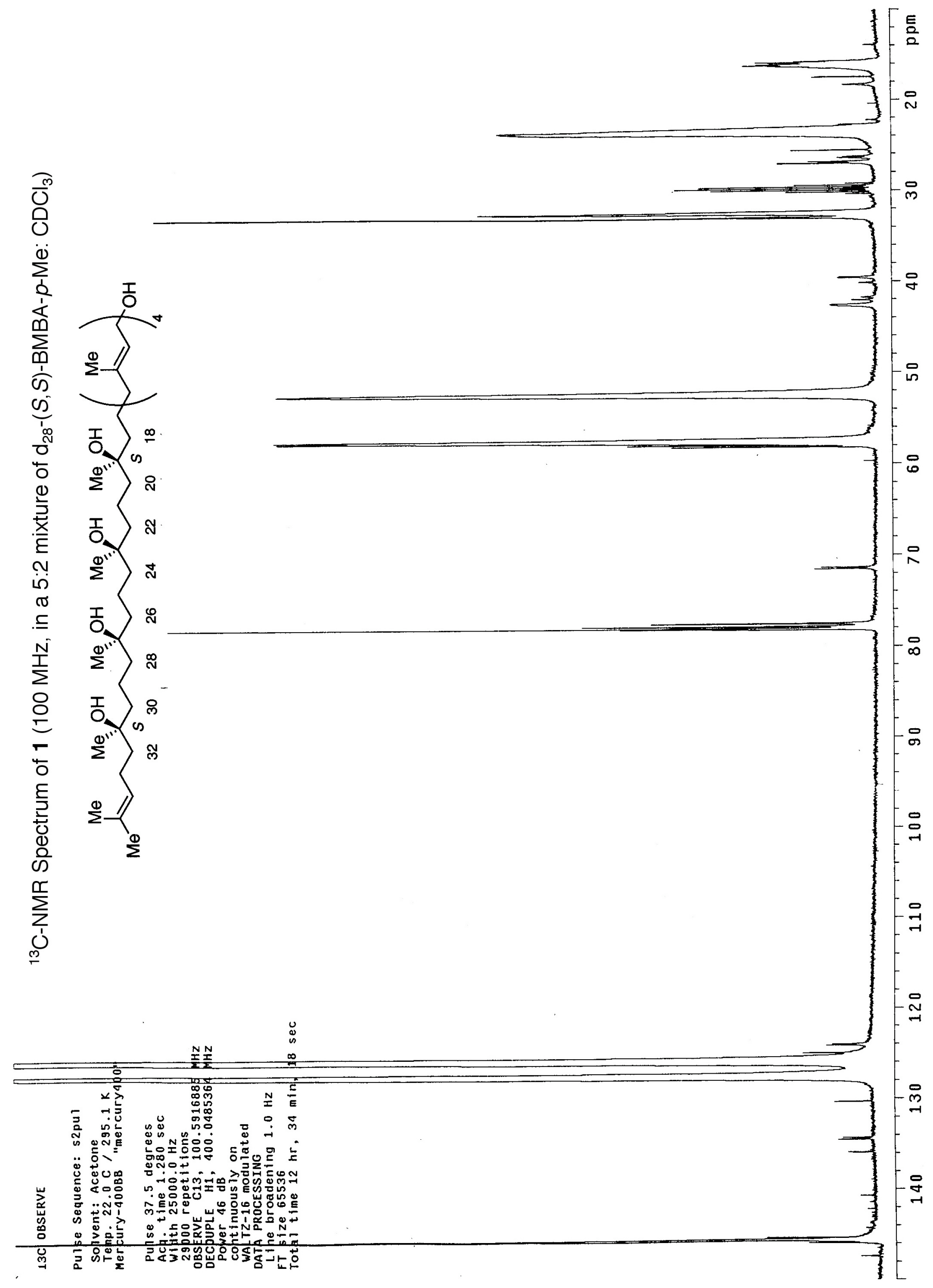



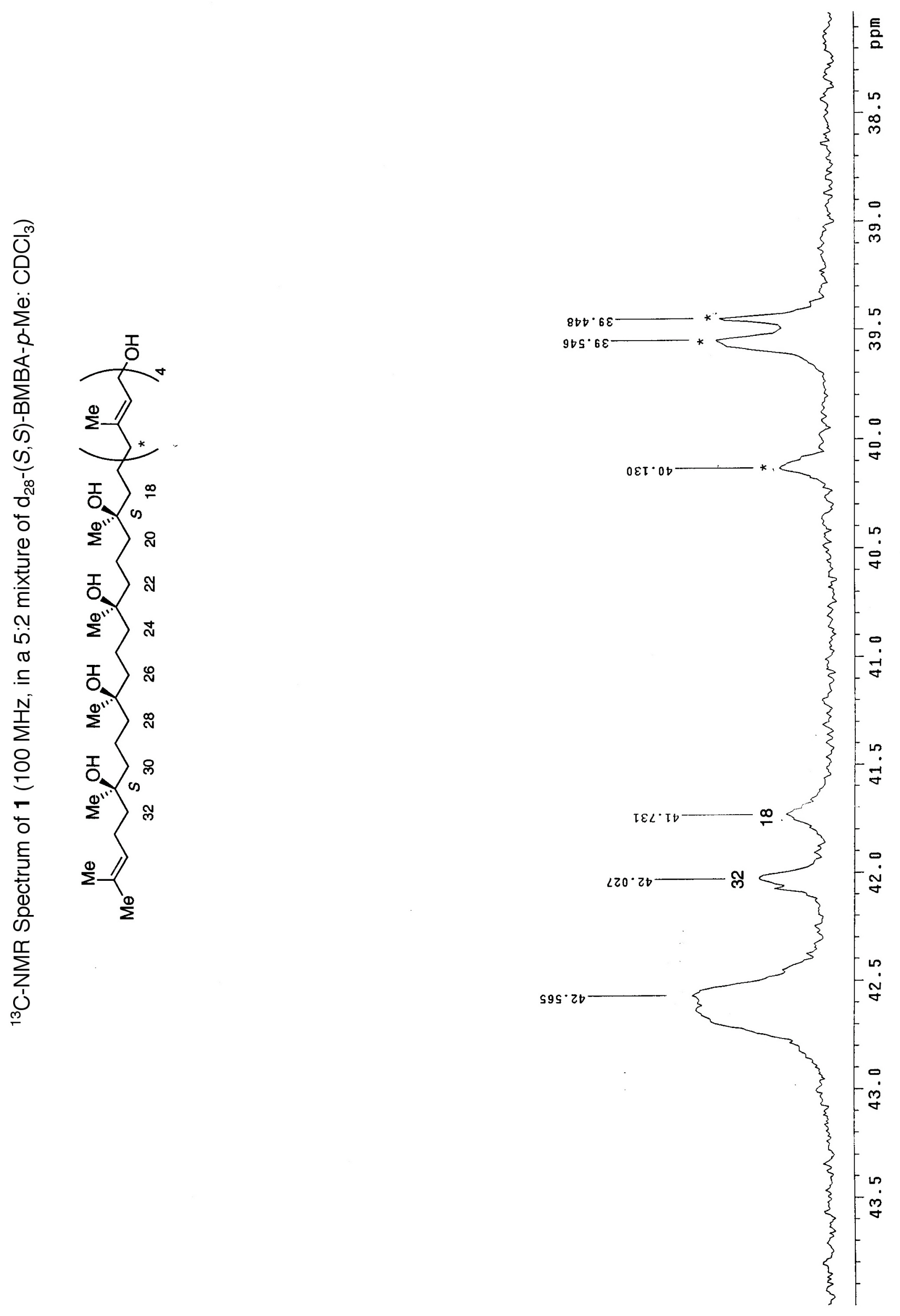


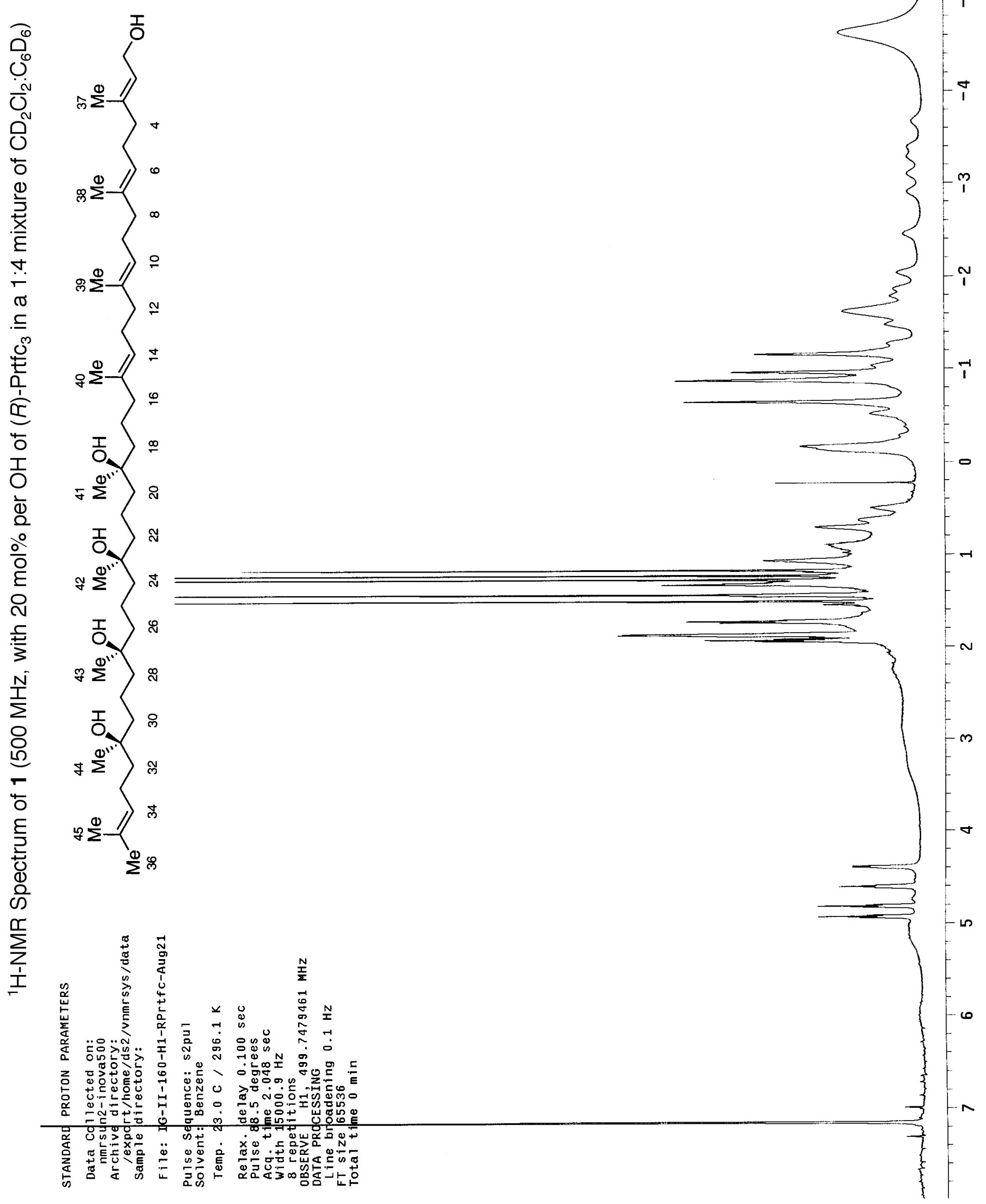



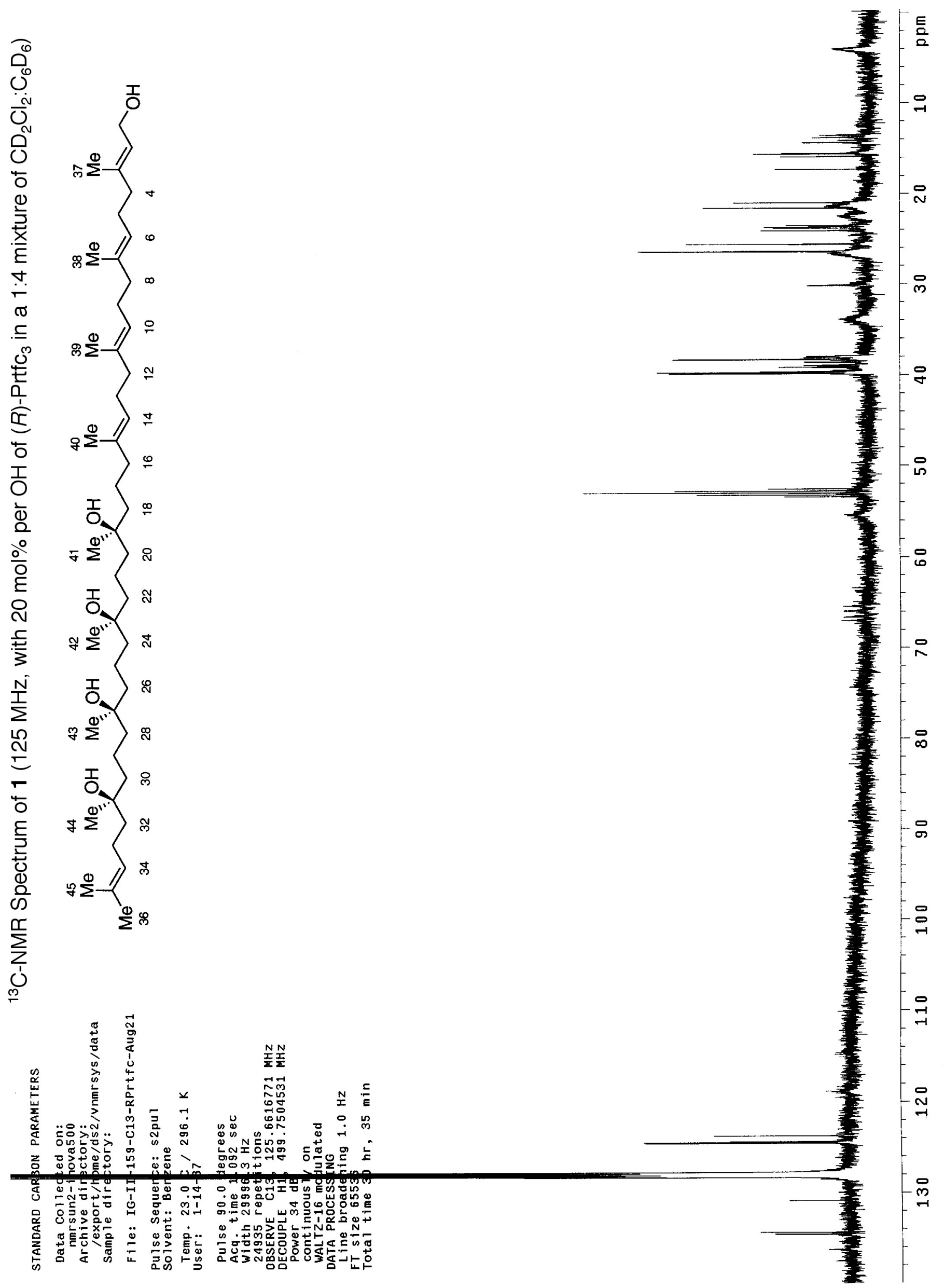

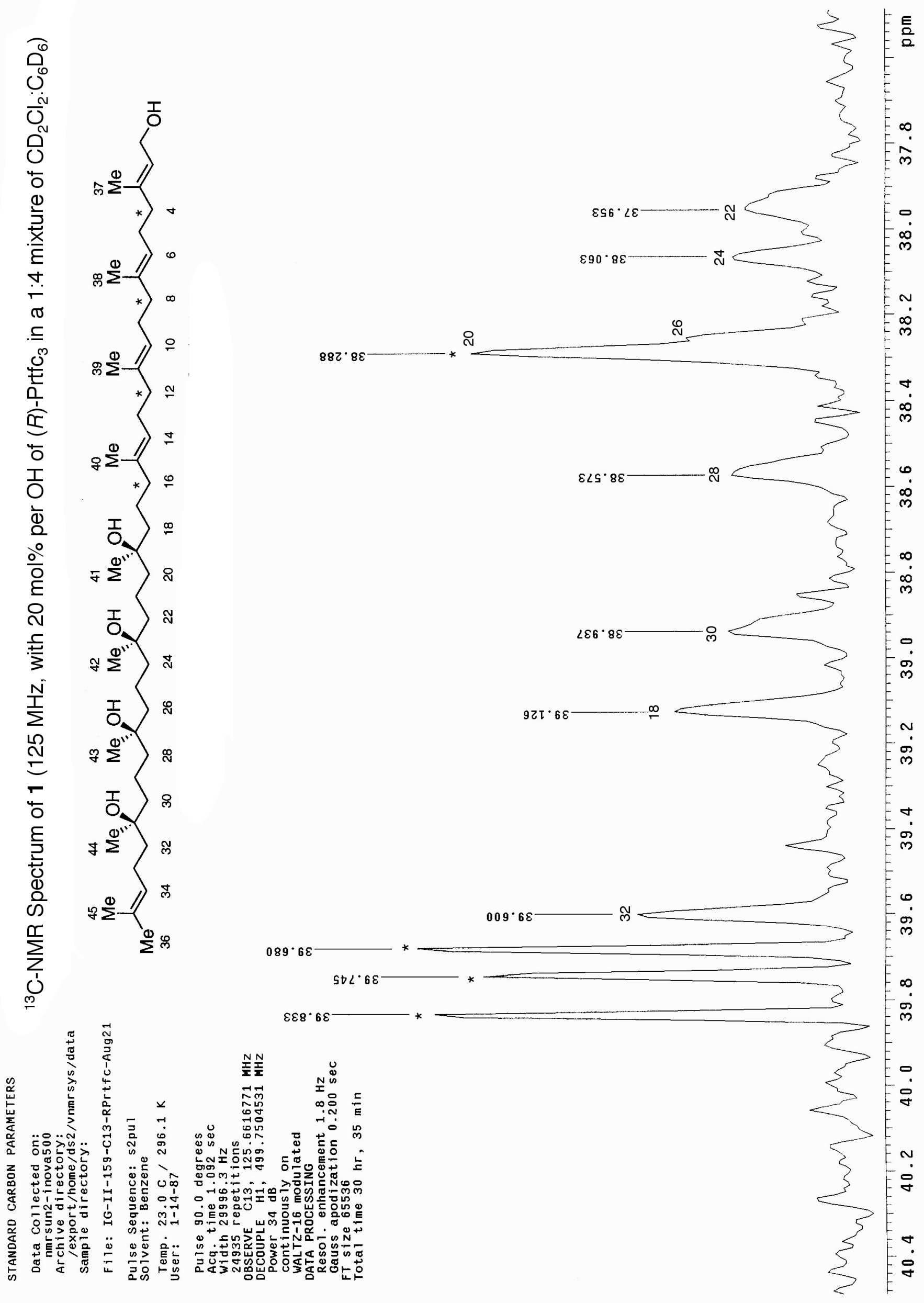


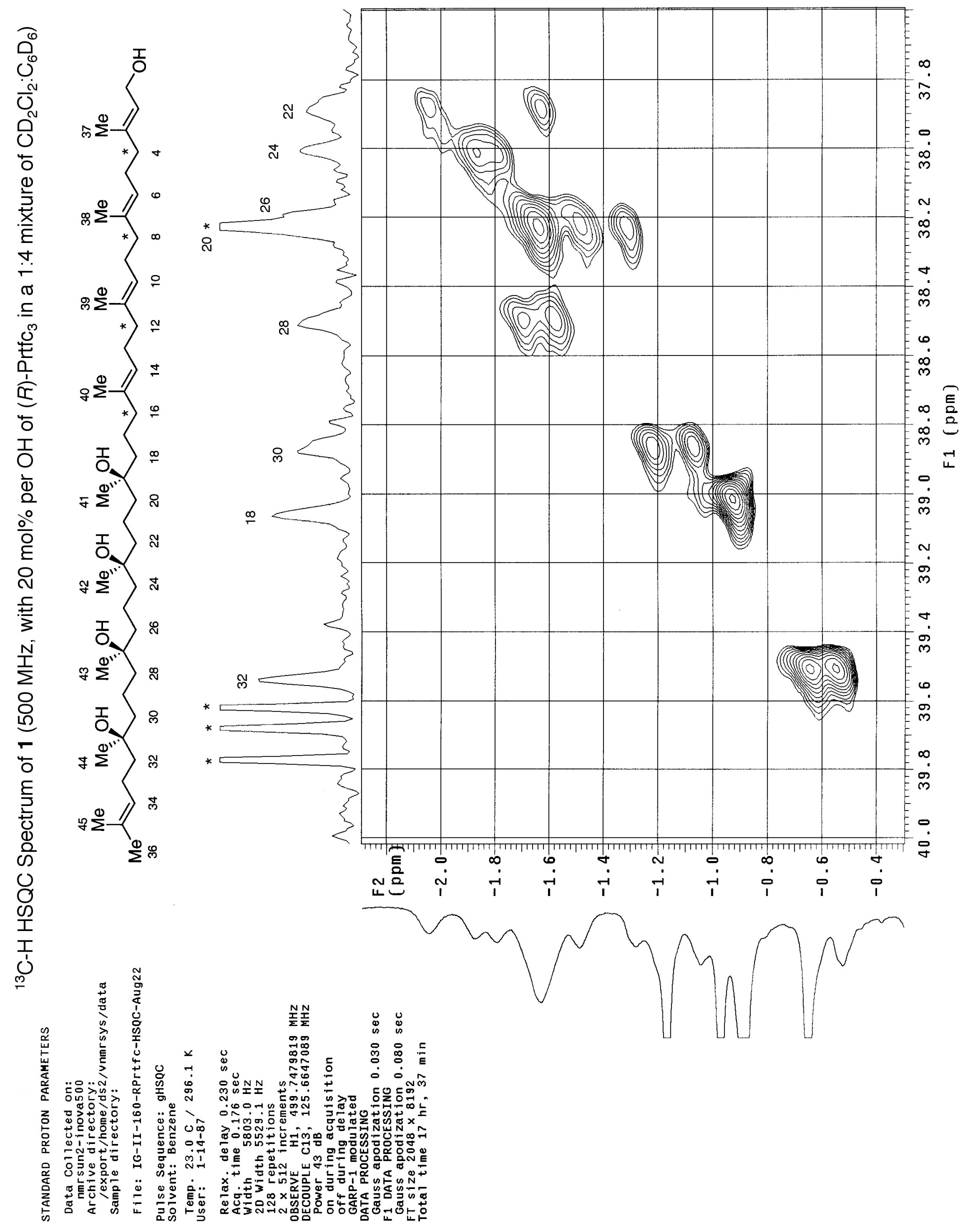




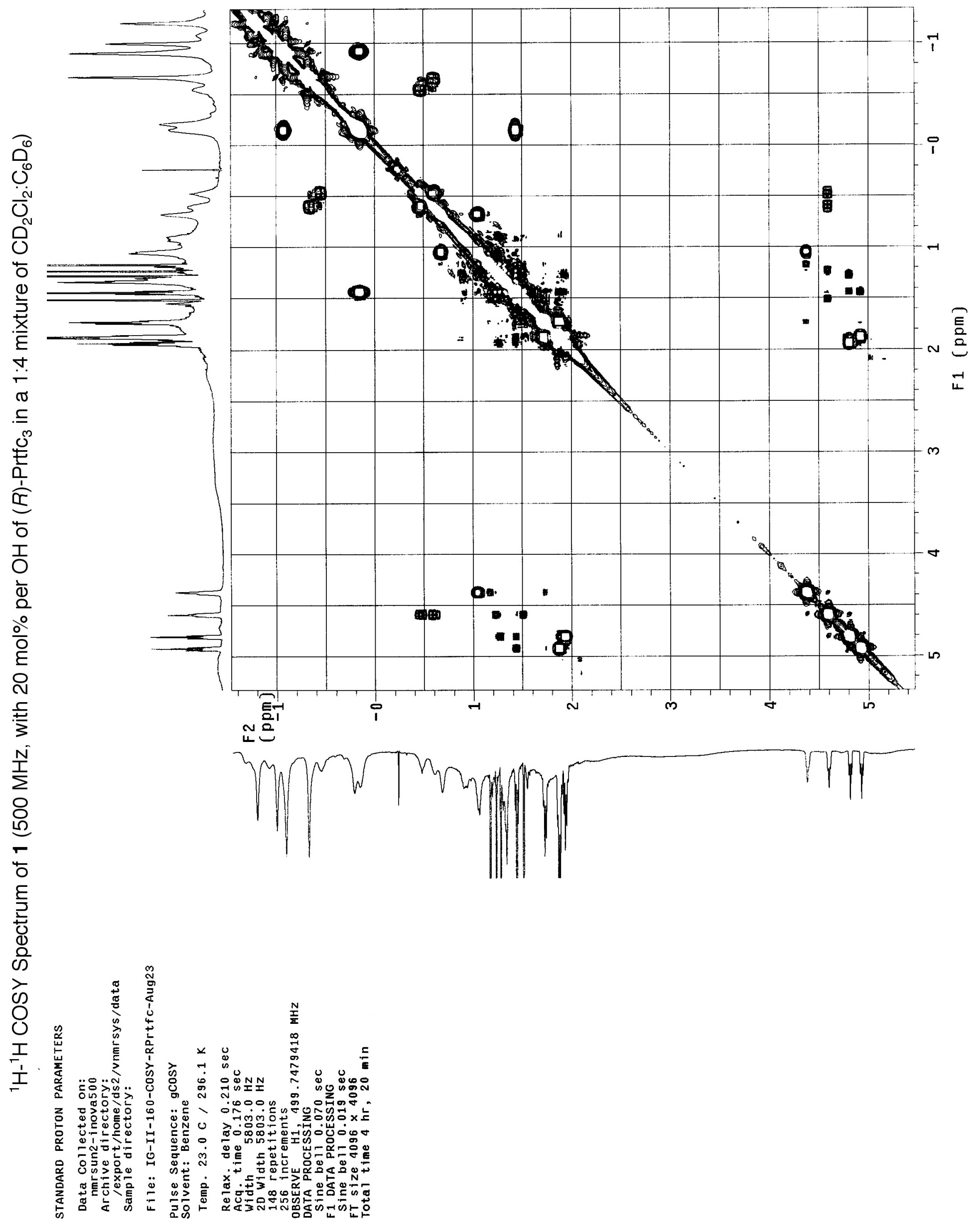




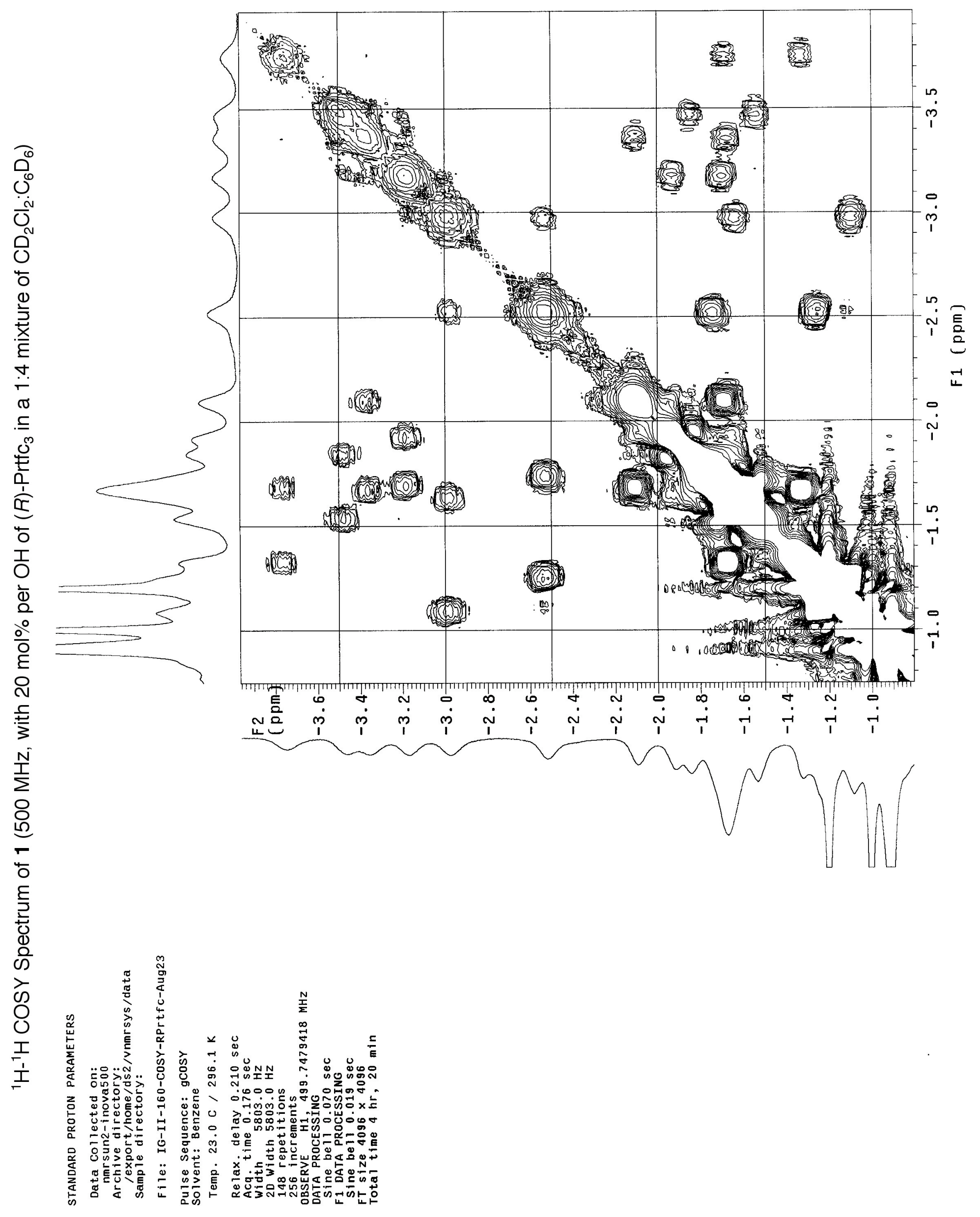




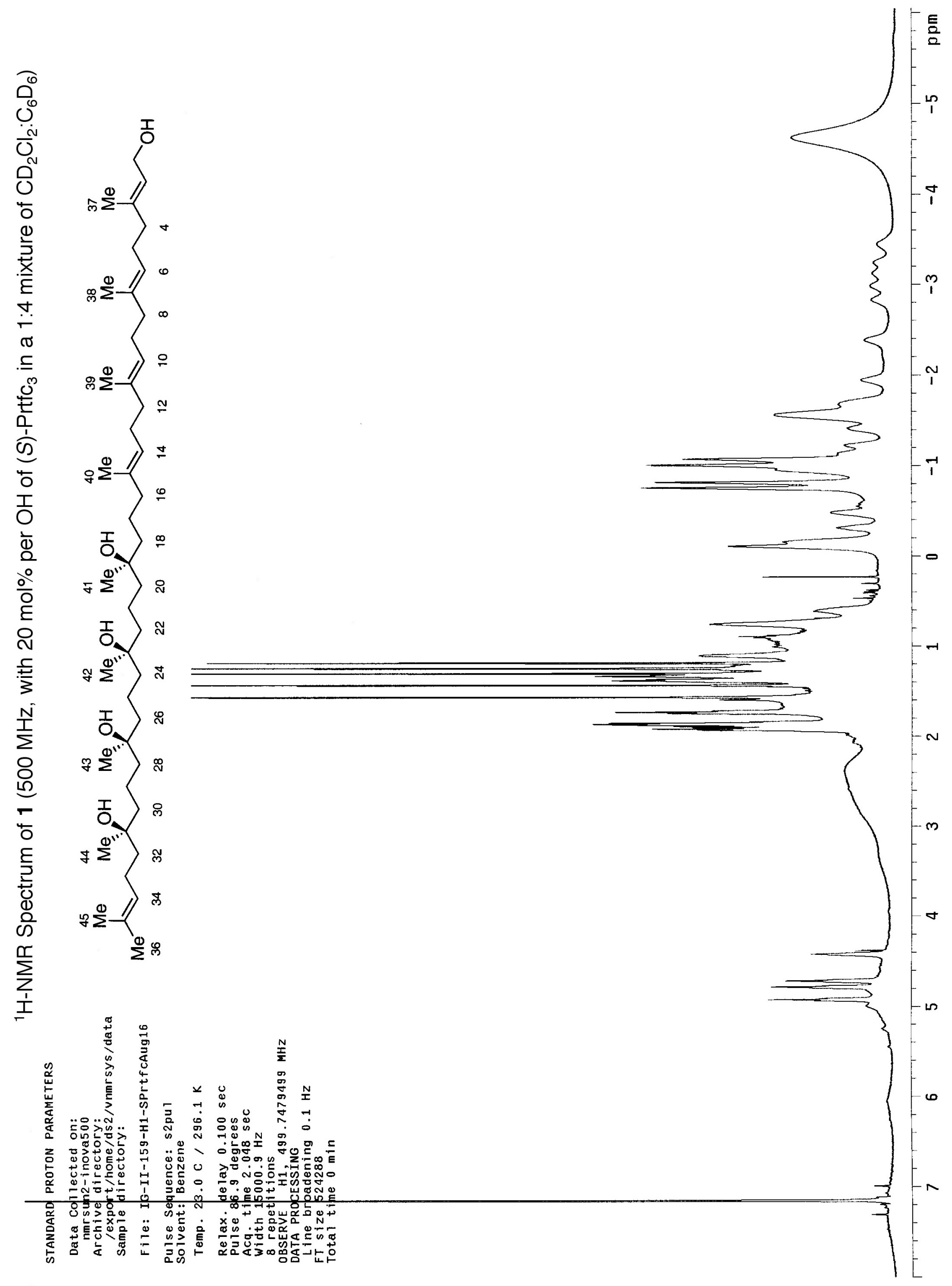




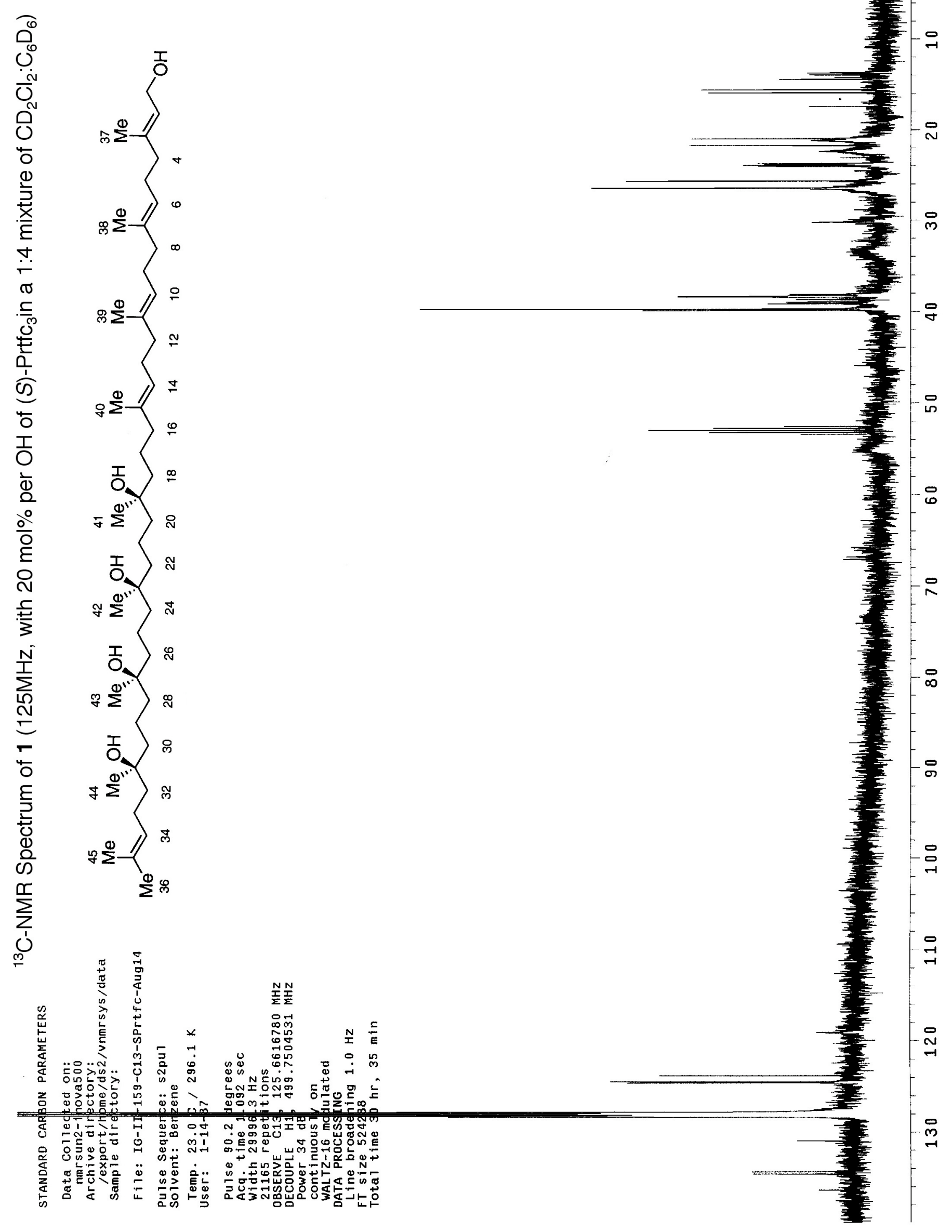



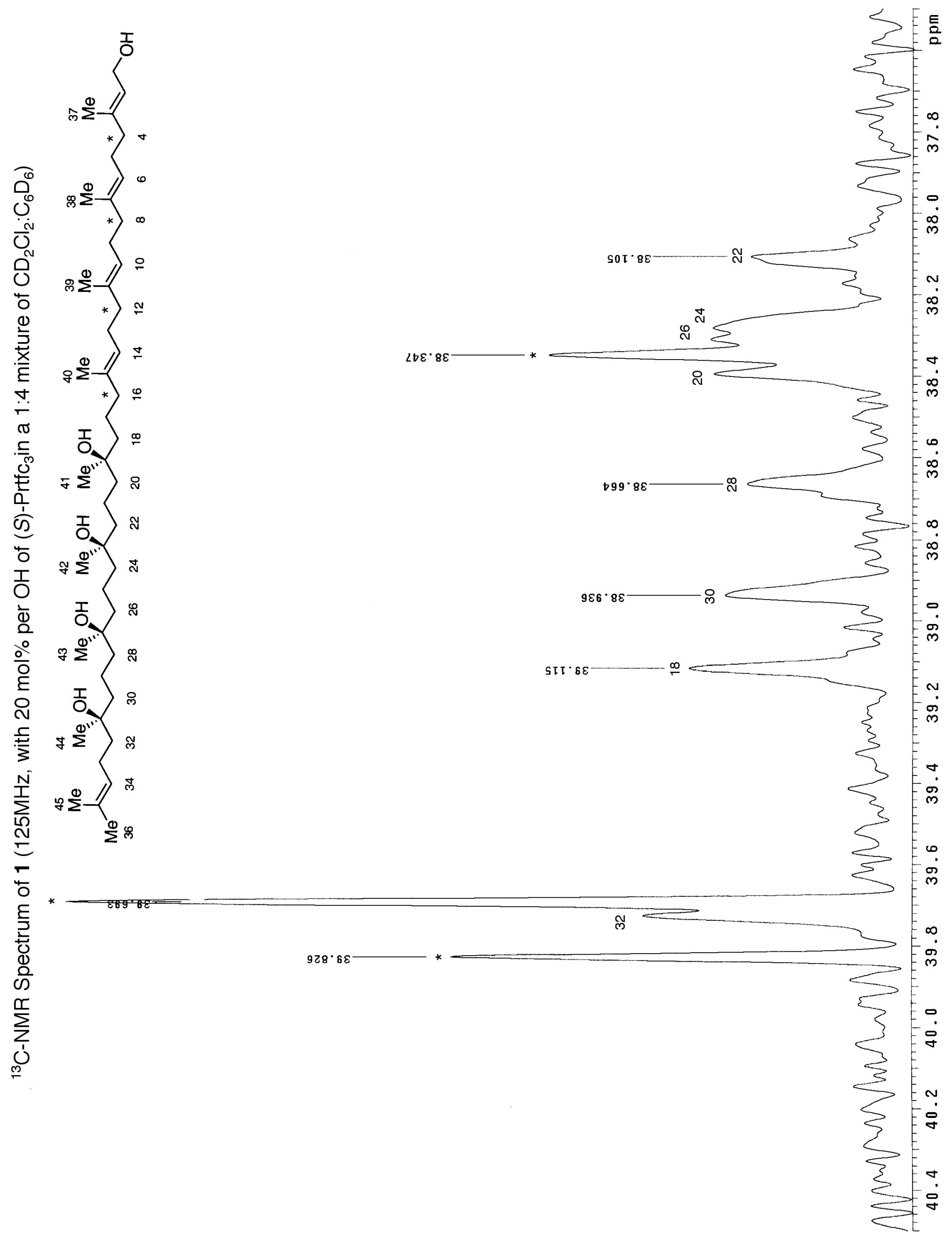


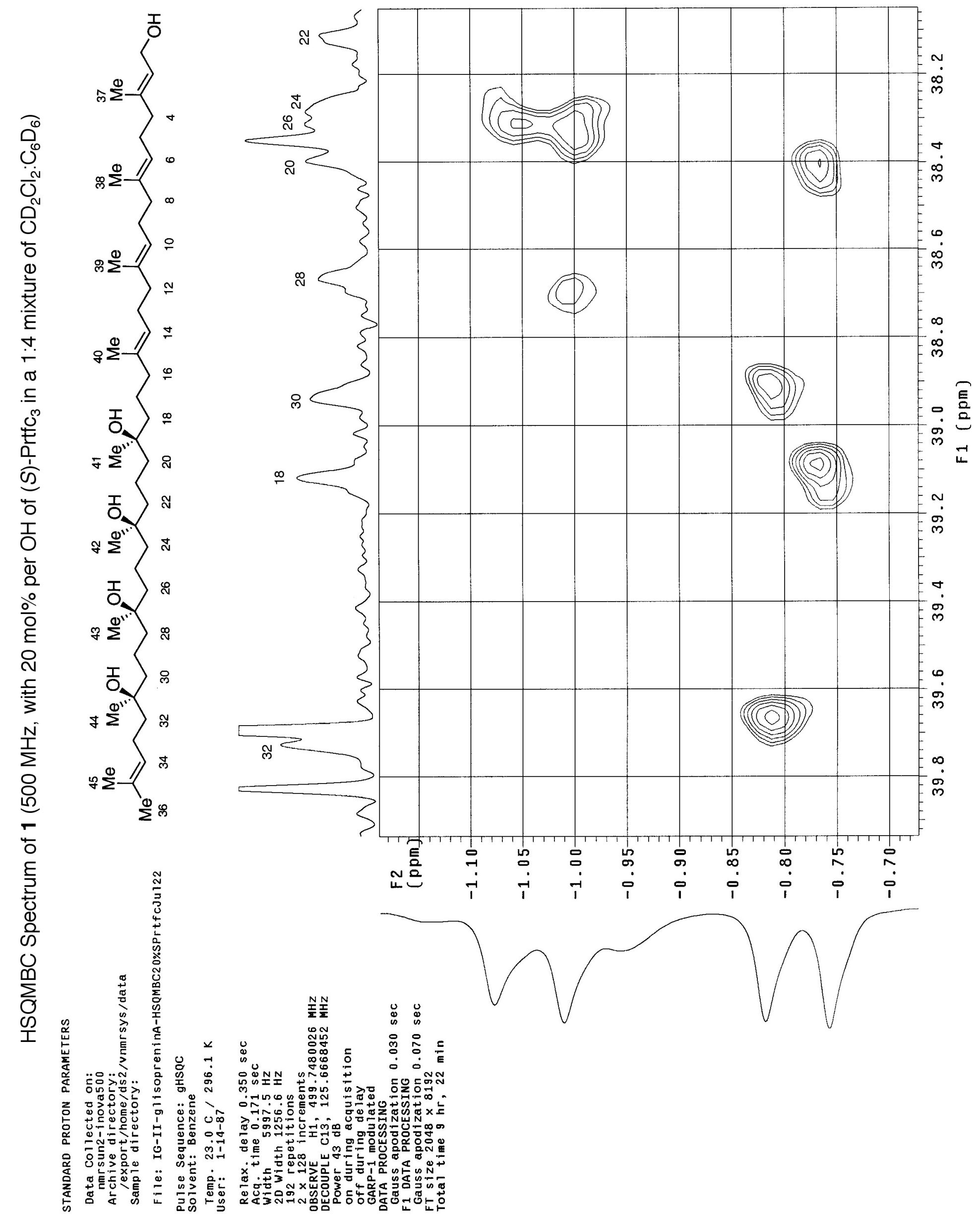




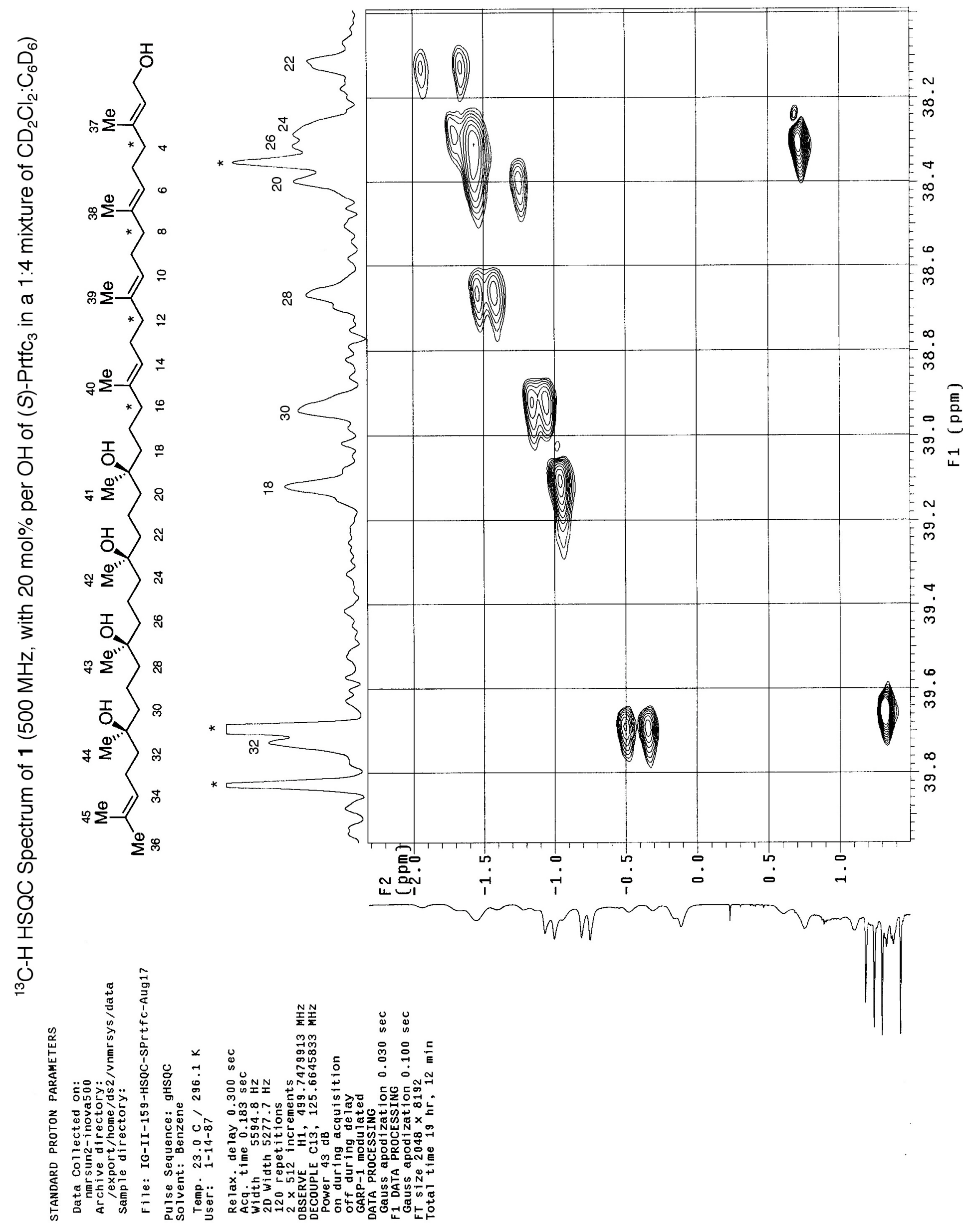



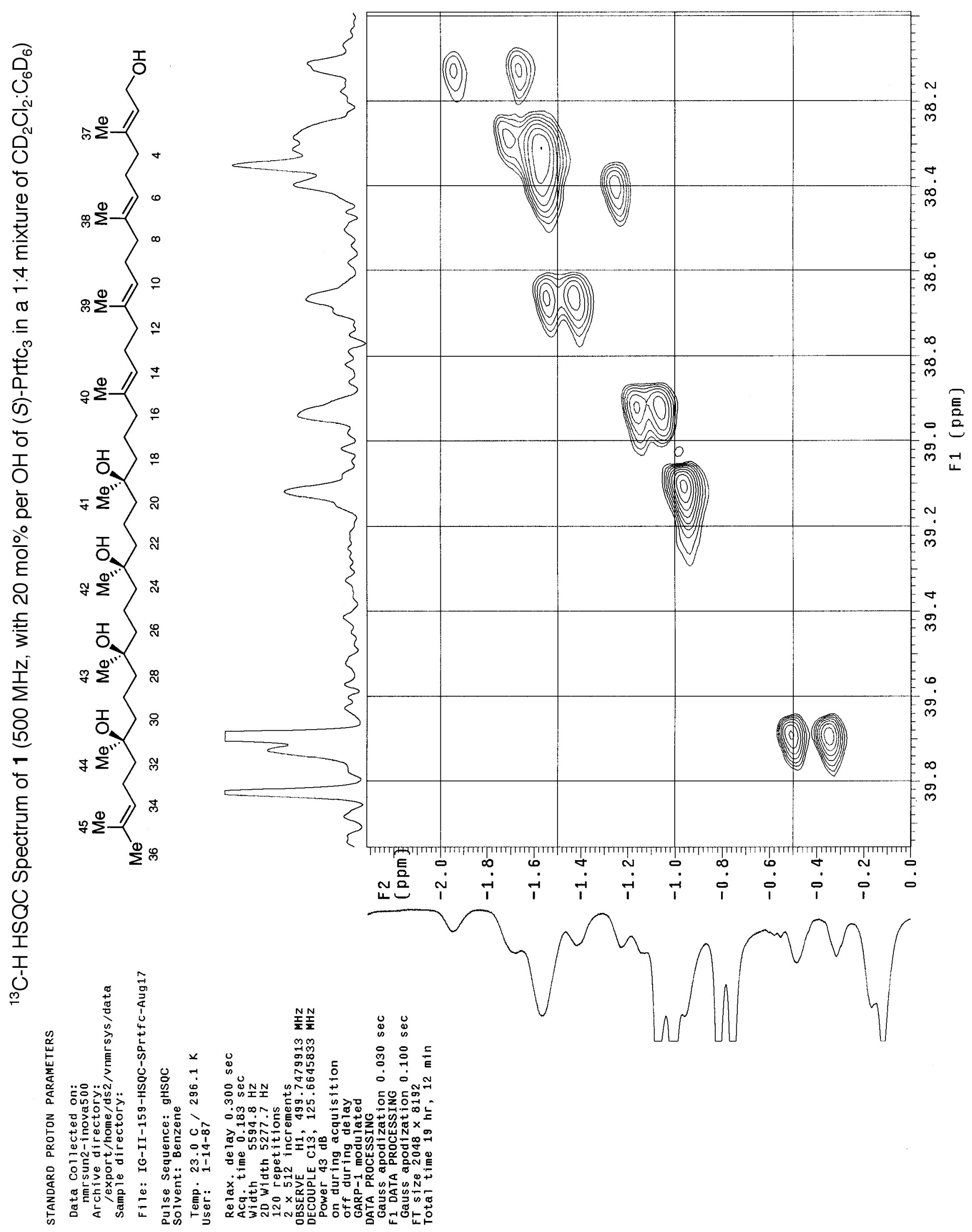


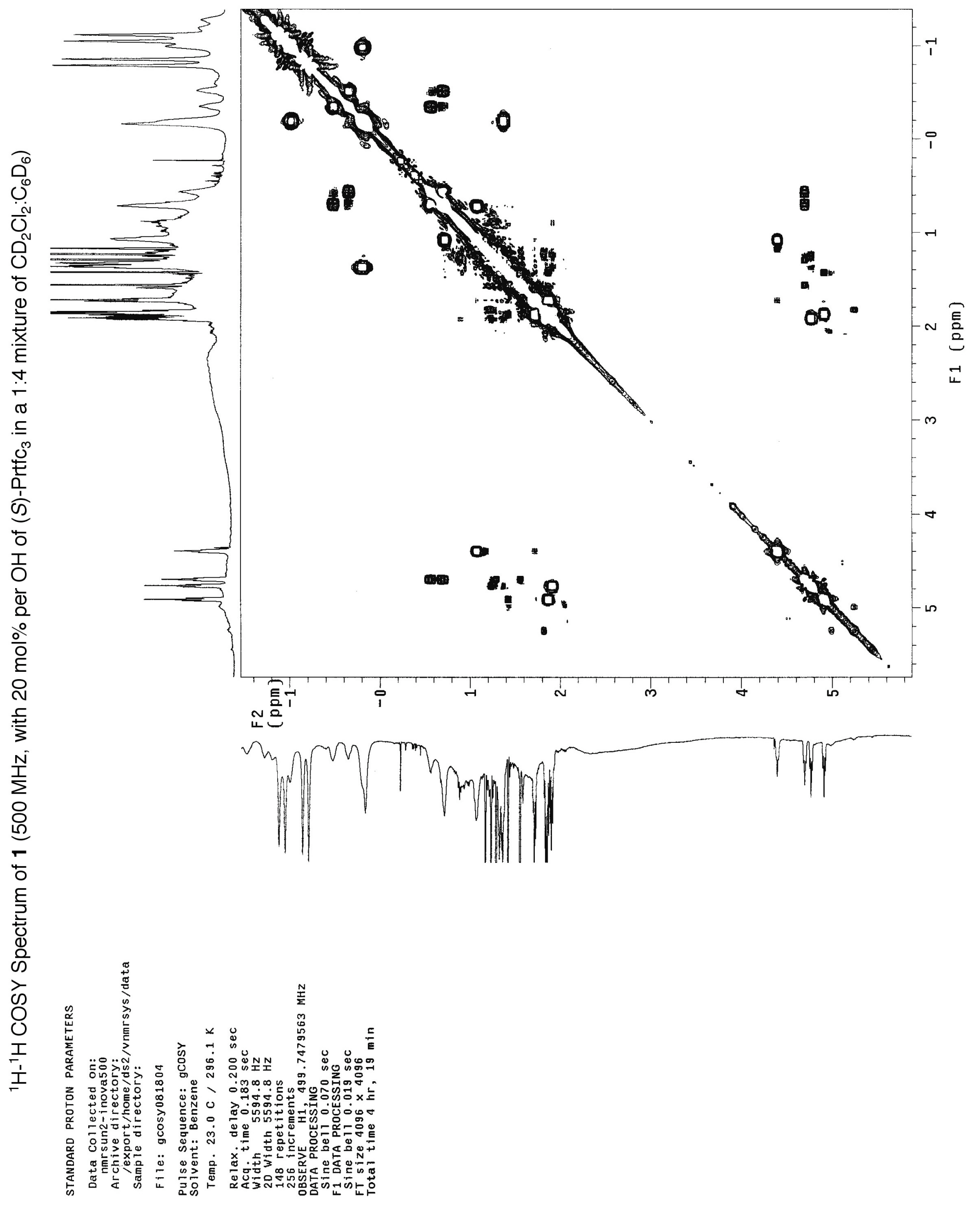




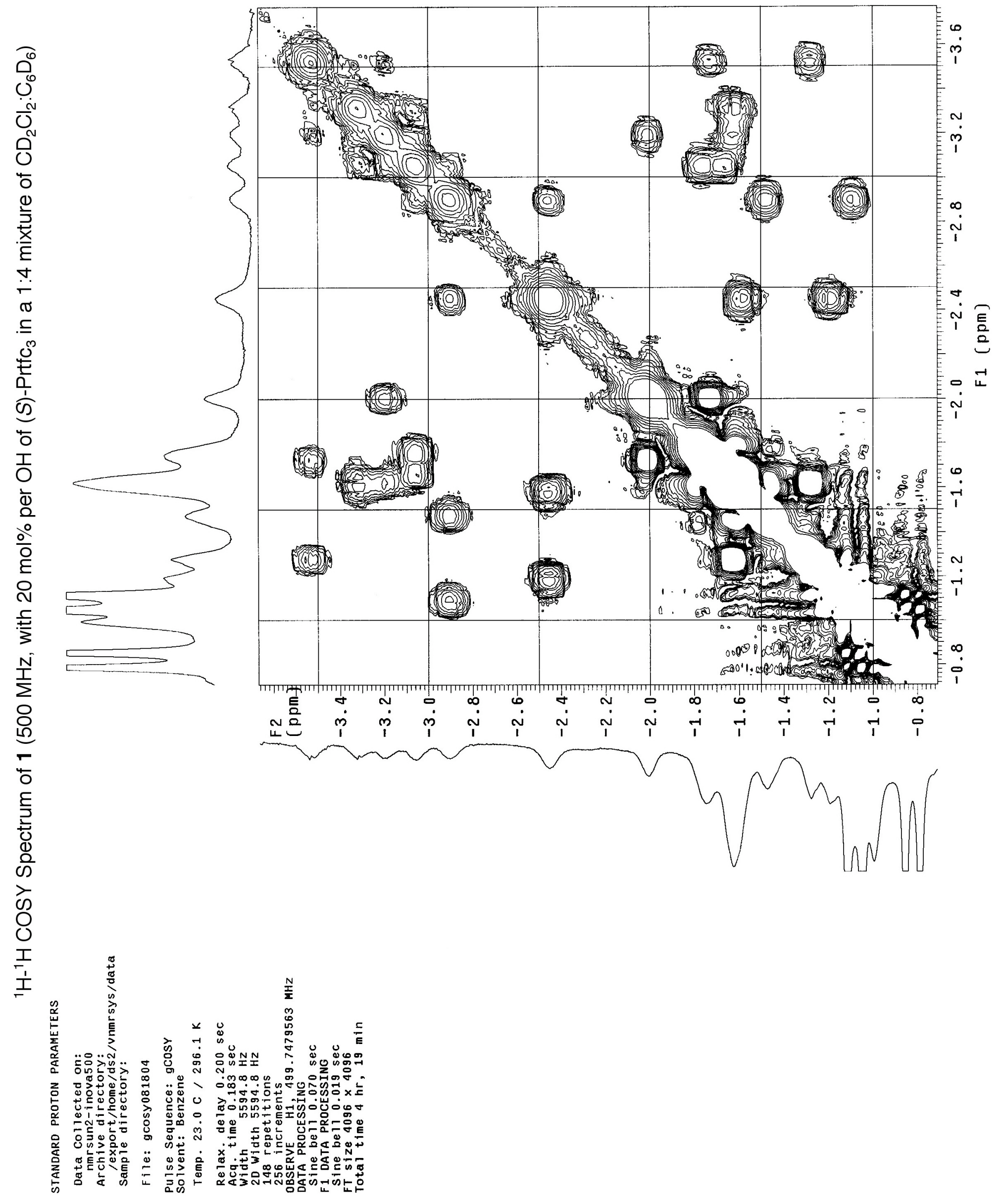

